\title{
Social network confidants, Duration of Untreated Psychosis and cannabis use in people with First Episode Psychosis: an exploratory study
}

\author{
Giuseppe Carrà ${ }^{\mathrm{a}, \mathrm{d}}$, Claudia Battaglia ${ }^{\mathrm{b}}$, Mark Hinton ${ }^{\mathrm{c}}$, \\ Luke Sheridan Rains $^{\mathrm{a}}$, Cristina Crocamo ${ }^{\mathrm{d}^{*}}$, Sonia Johnson ${ }^{\mathrm{a}, \mathrm{c}}$

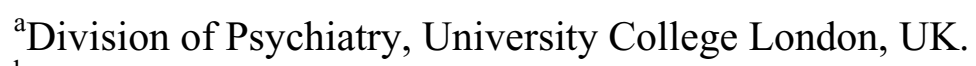 \\ ${ }^{b}$ Department of Neurosciences and Neurorehabilitation, Bambino Gesù Children's Hospital, Rome, \\ Italy \\ ${ }^{\mathrm{c}}$ Camden and Islington Early Intervention Service, London, UK \\ ${ }^{\mathrm{d}}$ Department of Medicine and Surgery, University of Milano-Bicocca, Italy.
}

Brief Report resubmitted for publication to Early Intervention in Psychiatry

Word count: Manuscript 1890; Abstract 148

Running Head: Social Network, DUP and cannabis

*Corresponding author: Department of Medicine and Surgery, University of Milano Bicocca. Via Cadore 48, 20900 Monza (MB), Italy. Tel. / Fax +390392332277. E-mail:

cristina.crocamo@unimib.it

\section{Funding and conflicts of interest}

None.

\section{Acknowledgments}

None.

This is the author manuscript accepted for publication and has undergone full peer review but has not been through the copyediting, typesetting, pagination and proofreading process, which may lead to differences between this version and the Version of Record. Please cite this article as doi: 10.1111/eip.12476

This article is protected by copyright. All rights reserved. 
Social network confidants, Duration of Untreated Psychosis and cannabis use in people with First Episode Psychosis: an exploratory study

Brief Report resubmitted for publication to Early Intervention in Psychiatry

Word count: Manuscript 1890; Abstract 148

Running Head: Social Network, DUP and cannabis

This article is protected by copyright. All rights reserved. 


\section{Abstract:}

Aim: Reduced social network (SN) might be associated with a longer Duration of Untreated Psychosis (DUP) in people with first episode psychosis (FEP). We aimed at exploring the specific role of SN confidants on DUP, taking into account cannabis misuse, which is known to influence DUP and might be associated with social functioning.

Methods: People with FEP recently referred to an inner London Early Intervention Service were assessed with standardized instruments exploring SN characteristics, DUP and cannabis misuse.

Results: Taking into account cannabis misuse, we found an association between confidants and DUP ( $p=0.020)$, with the higher the number of confidants, the shorter the DUP.

Conclusions: Confidants may provide access to, and perceptions of, social support and this may increase early engagement for people with FEP, reducing DUP and possibly improving outcomes. Future research should identify correlates of small networks of confidants, which could inform early detection community initiatives.

Key words: cannabis, DUP, first episode psychosis, social network, social support 


\section{INTRODUCTION}

People with long-standing psychotic disorders have fewer social contacts and less social support than comparison groups (e.g., ${ }^{1}$ ). They frequently have difficulties in developing and maintaining social relationships, reporting smaller social networks than those from nonclinical populations and networks that are near exclusively composed of family members ${ }^{2}$. This is also true for young people with first episode psychosis (FEP) who report reduced social networks (particularly for close friends) and lower support, though this seems to predate onset of psychotic disorders ${ }^{3}$. In addition, if friends are separately considered as confidants and acquaintances, this brings even stronger evidence about the paucity of confidants for people with FEP ${ }^{4}$.

Unfortunately, a number of inconsistencies have hampered progress in understanding the significance of Social Networks (SN) research so far. First, research to date is almost invariably cross-sectional which has challenged efforts to disentangle fundamental questions including whether poor social networks among people with FEP simply belong to the complex cluster of causes leading to psychosis or whether they are a consequence of prodromal stages and onset of the disorder. The limited longitudinal literature shows contrasting findings. A reduction in social networks following onset was reported in early literature ${ }^{5}$, not replicated in later studies ${ }^{6}$. A second methodological issue that diminishes our understanding of the impact of social networks on the onset and the early course of psychosis involves the wide range of measures used, posing challenges in comparing findings and drawing conclusions. For example, when comparing size of networks some studies have used detailed frameworks specifically naming everyone known to participants (e.g., ${ }^{5}$ ), while others have counted only those people who are considered 'close' connections ${ }^{7}$ or those people with whom participants have had contact in a prescribed time frame (e.g., the last month $)^{6}$. Thus, analyses considering the total number of contacts collected may be misleading 
${ }^{8}$. At the same time, few studies have adjusted for alternative variables that might explain the observed associations between social networks and FEP ${ }^{4}$. Several studies have found that a longer duration of untreated psychosis (DUP), which predicts poor clinical outcomes in FEP ${ }^{9}$, is associated with a low frequency of social contacts $\left(\right.$ e.g., $\left.{ }^{10}\right)$. While this was not confirmed in other studies, a role for unemployment, poor academic performances ${ }^{11,12}$, positive and negative symptoms levels ${ }^{13}$, social withdrawal and isolation, and poorer global and premorbid functioning ${ }^{14}$ have all been identified as contributing to reduced social networks and support.

More importantly, cannabis use seems an important candidate variable to consider. Recent, high and frequent cannabis use seems to reduce DUP and is associated with accelerated transition to psychosis ${ }^{15-17}$. However, cannabis-using subjects have higher cognitive and social functioning, compared to non-users ${ }^{18}$, possibly because the demands of their cannabis use dictate that the individual needs to engage with drug- dealers and others in order to secure supply ${ }^{19}$, which might influence their social networks size and composition.

We argue the literature thus far has not fully explored the potential confounding role of cannabis use on the relationship between social network and DUP ${ }^{3}$. The present study was designed to test the hypothesis that people with FEP may have a shorter DUP if they have a larger social network particularly in terms of close confidants, and taking into account the confounding role of cannabis use.

\section{METHODS}

\section{Participants}

This naturalistic study aimed to include all consecutive referrals to the Camden \& Islington NHS Foundation Trust, Early Intervention Service (EIS). Inclusion, and service eligibility, 
criteria were: (i) aged between 18 and 35 years old; (ii) presenting to the EIS for the first time for affective or non-affective psychosis; and (iii) resident within the EIS catchment area. The local research ethics committee provided ethical permission. All service users who were accessible and stable were approached for informed, signed, consent several months after service entry.

\section{Assessment measures}

Routine sociodemographic data were collected. The Social Network Schedule (SNS), a selfreport instrument was used to assess all social contacts during the preceding month ${ }^{20}$. Contacts might include staff, patients, relatives, social acquaintances, neighbours, workmates, service contacts and other. Once a list of all contacts is established, SNS explores several domains about each of them, including confidant status, frequency of contact with participants (daily, weekly, monthly), and whether the person considered a contact would be missed in case if he/she should be lost and whether relationships were characterized by upset and conflict. Duration of untreated psychosis was assessed by the Nottingham Onset Schedule-DUP version ${ }^{21}$, a widely used tool whose reliability and validity are quantitatively comparable to other DUP measurement instruments ${ }^{22}$. The DUP measure of interest was the interval (months) between onset of the prodrome and the start of anti-psychotic medication. Information on current symptom profile was collected through the 30-item Positive and Negative Syndrome Scale (PANSS ${ }^{23}$ ). The Global assessment for symptoms (GAF-S) and disability (GAF-D) scales were used to assess functioning in various domains associated with social interactions ${ }^{24}$. Finally, the alcohol and cannabis use of participants was assessed with the Drake Clinician Rating Scales for Alcohol (AUS) and Drug (DUS) Use ${ }^{25}$, as appropriate for people with comorbid misuse and psychosis, not necessarily requiring more time consuming structured interviews in terms of reliability and validity ${ }^{26,27}$.

\section{Statistical analysis}


Analyses were carried out using Stata (13.1; Stata Corp, College Station, Texas). Level of significance was set at 5\%, and all p-values were two-tailed. In order to explore the specific role of SN confidants on DUP, we first examined the distribution of DUP, including residuals' distribution showing whether the conditional expected response was linear in the fitted values. Thus, we checked on normality of residuals' distribution (quantiles of residuals against quantiles of normal distribution). In addition, we verified the opportunity to apply data transformation (e.g., logarithmic), using the ladder of powers for a transform that converts the original variable into a normally distributed variable ${ }^{28}$.

We also took into account other variables, including cannabis misuse, which could influence DUP and might be associated with social functioning. We thus examined correlates of DUP and SNS measures. Along with non-parametric Mann-Whitney test for nominal independent variables, the non-parametric Spearman's Rho test was used to calculate bivariate correlations, since relevant distributions were positively skewed. Thereafter, a multiple linear regression model was used to assess the strength of the relationship between number of confidants as proxy for social network support and DUP as dependent variable, controlling for potential confounders.

Table 1 about here

\section{RESULTS}

One hundred and twenty-two people with FEP were included in the study. Sociodemographic, clinical and social network characteristics are shown in Table 1 . The majority of subjects were men, with mean age and DUP of $24.2(\mathrm{SD}=4.2)$ years and $9.2(\mathrm{SD}=11.9)$ months, respectively. More than a half reported cannabis use, whilst $76 \%$ of alcohol. Functioning showed mean scores of $36.7(\mathrm{SD}=16.9)$ on GAF-S and of $45.3(\mathrm{SD}=17.1)$ on GAF-D, whilst average positive and negative symptoms profiles on PANSS were 22.7 
$(\mathrm{SD}=6.4)$ and $18.5(\mathrm{SD}=8.6)$. Although mean total contacts were $11(\mathrm{SD}=15.5$, median 7), mean numbers of confidants were reported as low as five $(\mathrm{SD}=4.0$, median $=5)$. Interestingly, the number of contacts that would be missed was on average higher than of those with whom participants were in conflict. Most contacts were met with a frequency of between 3/4 days per week and weekly.

Further correlational analyses were conducted identifying relevant variables in relation to DUP and number of confidants. DUP was correlated with the number of confidants $(r=-$ $0.25, p=0.007)$ and PANSS positive $(r=-0.33 \mathrm{p}=0.0004)$, and associated with cannabis misuse $(p=0.025)$. No statistically significant correlations were detected with age, years of education, GAF-S and GAF-D, and PANSS negative. On the other hand, the number of confidants was associated with cannabis misuse $(\mathrm{p}=0.024)$ and was negatively correlated with age $(r=-0.25, p=0.006)$, GAF-S score $(r=-0.24, p=0.013)$ as well as with DUP. Moreover, albeit gender may play a key role in terms of social networks and clinical characteristics ${ }^{29}$, our data did not show any statistically significant differences, even if both DUP and number of confidants were slightly higher among women. Then, we evaluated the relationship between the number of confidants and DUP, using a log transformed DUP in an unadjusted linear regression model. A decrease of $9.8 \%$ in DUP $(p=0.01)$ was observed for a one unit increase in number of confidants. However, we had to take into account potential confounding, considering characteristics associated with both DUP and the number of confidants at univariate level, i.e., cannabis misuse. Thus, considering potential confounding, a multiple linear regression model examining the relationship between the number of confidants and DUP, controlling for cannabis misuse (Table 2), showed a significant, negative, correlation between the number of confidants and DUP, with the higher the number of confidants, the shorter the DUP. This effect, though of limited magnitude, remained 
virtually unmodified also taking into account cannabis misuse $(8.9 \%$ decrease in DUP for a one-unit increase in the number of confidants while cannabis misuse held constant).

Table 2 about here

\section{DISCUSSION}

This naturalistic study of FEP people in London, UK, found that a higher number of confidants in their social network was associated with a shorter DUP. This was also true after considering cannabis use, though this may relate to the need to secure supplies, interacting with misusing peers ${ }^{27}$. A better understanding of specific social networks related to cannabis misuse is needed, given its association with transition to psychosis ${ }^{30}$. Our findings about the association between paucity of confidants with a longer DUP are consistent with previous research $^{4,11,29}$, even discounting total social network size ${ }^{6}$. However, a wider social network does not necessarily imply this is always activated and used in relation to specific stressors, which are typical during early stages of psychosis ${ }^{3}$. Confidants may provide access to, and perceptions of, social support and this might increase early engagement with EIS, reducing DUP and thus improving outcomes ${ }^{9}$.

We acknowledge several limitations. Along with the cross-sectional nature of our study, that does not allow making any inference on causality, it might well be that the limited number of confidants, assessed by SNS in the preceding month, is simply a consequence of prodromal stages and onset. In addition, we could detect only one confounding factor (i.e., cannabis misuse), but several others could affect the results of our study. Thus, it would be important that future research would identify alternative confounding elements, also benefiting of larger samples, such as the role of family members ${ }^{31}$, of stigma ${ }^{32,33}$, and of the organization of mental health services ${ }^{34}$. 
Moreover, our sample was drawn from an inner-city, ethnically diverse, population in the UK and thus the results may not be generalizable to EIS in rural locations or other countries. In addition, assessment and data stream by self-report approach might result in unreliable and incomplete data with a poor ability to distinguish participants in a more effective way, typical of non self-report measures.

Clinical services can exploit these findings considering that the nature of relationships of people with FEP with confidants is based on intimacy, commitment, and interaction, perhaps also including confidants' social connections into their own network ${ }^{35}$. These domains may appear beyond the scope of clinical services ${ }^{36}$. However, if future research could identify correlates of smaller network of confidants, this could inform early detection initiatives at a local level ${ }^{37}$, possibly developing more social ties in their communities. 


\section{References}

1. Palumbo C, Volpe U, Matanov A, Priebe S, Giacco D. Social networks of patients with psychosis: a systematic review. BMC Res Notes 2015; 8: 560.

2. Buchanan J. Social support and schizophrenia: a review of the literature. Arch Psychiatr Nurs 1995; 9: 68-76.

3. Gayer-Anderson C, Morgan C. Social networks, support and early psychosis: a systematic review. Epidemiol Psychiatr Sci 2013; 22: 131-46.

4. Morgan C, Kirkbride J, Hutchingson G, et al. Cumulative social disadvantage, ethnicity and first-episode psychosis: a case-control study. Psychol Med 2008; 38: 1701-15.

5. Lipton FR, Cohen CI, Fischer E, Katz SE. Schizophrenia: a network crisis. Schizophr Bull 1981; 7: 144-51.

6. Thorup A, Petersen L, Jeppesen P, et al. Social network among young adults with firstepisode schizophrenia spectrum disorders: results from the Danish OPUS trial. Soc Psychiatry Psychiatr Epidemiol 2006; 41: 761-70.

7. Erickson DH, Beiser M, Iacono WG, Fleming JA, Lin TY. The role of social relationships in the course of first-episode schizophrenia and affective psychosis. Am J Psychiatry 1989; 146: 1456-61.

8. Horan WP, Subotnik KL, Snyder KS, Nuechterlein KH. Do recent-onset schizophrenia patients experience a "social network crisis"? Psychiatry 2006; 69: 115-29.

9. Penttilä M, Jääskeläinen E, Hirvonen N, Isohanni M, Miettunen J. Duration of untreated psychosis as predictor of long-term outcome in schizophrenia: systematic review and meta-analysis. Br J Psychiatry 2014; 205: 88-94.

10. Kalla O, Aaltonen J, Wahlstrom J, et al. Duration of untreated psychosis and its correlates first-episode psychosis in Finland and Spain. Acta Psychiatr Scand 2002; 106: $265-75$.

This article is protected by copyright. All rights reserved. 
11. Reininghaus UA, Morgan C, Simpson J, et al. Unemployment, social isolation, achievement-expectation mismatch and psychosis: findings from the AESOP study. Soc Psychiatry Psychiatr Epidemiol 2008; 43: 743-51.

12. Peralta V, Cuesta MJ, Martinez-Larrea A, Serrano JF, Langarica M. Duration of untreated psychotic illness: the role of premorbid social support networks. Soc Psychiatry Psychiatr Epidemiol 2005; 40: 345-9.

13. Devylder JE, Gearing RE. Declining social support in adolescents prior to first episode psychosis: associations with negative and affective symptoms. Psychiatry Res 2013; 210: $50-4$.

14. Hui CL, Lau WW, Leung CM, et al. Clinical and social correlates of duration of untreated psychosis among adult-onset psychosis in Hong Kong Chinese: the JCEP study. Early Interv Psychiatry 2015; 9: 118-25.

15. Burns JK. Cannabis use and duration of untreated psychosis: a systematic review and meta-analysis. Curr Pharm Des 2012; 18: 5093-104.

16. Dominguez MD, Fisher HL, Major B, et al. Duration of untreated psychosis in adolescents: ethnic differences and clinical profiles. Schizophr Res 2013; 150: 526-32.

17. Valmaggia LR, Day FL, Jones C, et al. Cannabis use and transition to psychosis in people at ultra-high risk. Psychol Med 2014; 44: 2503-12.

18. Potvin S, Stavro K, Pelletier J. Paradoxical Cognitive Capacities in Dual Diagnosis Schizophrenia: The Quest for Explanatory Factors. Journal of Dual Diagnosis 2012; 8: $35-47$.

19. Salyers MP, Mueser KT. Social functioning, psychopathology, and medication side effects in relation to substance use and abuse in schizophrenia. Schizophr Res 2001; 48: 109-23. 
20. Dunn M, O’Driscoll C, Dayson D, Will W, Leff J. The TAPS project. 4: an observational study of long-stay patients. Br J Psychiatry 1990; 157: 842-8, 852.

21. Singh SP, Cooper JE, Tarrant CJ, Bagalkote H, Jones PB. Establishing onset in first episode psychosis: The Nottingham onset schedule (NOS). European Psychiatry 2000; 15: s283-4.

22. Register-Brown K, Hong LE. Reliability and validity of methods for measuring the duration of untreated psychosis: a quantitative review and meta-analysis. Schizophr Res. 2014; 160: 20-6.

23. Norman RMG, Malla AK, Cortese L, Diaz F. A study of the inter- relationship between and comparative interrater reliability of the SAPS, SANS and PANSS. Schizophr Res 1996; 19: 73-85.

24. Endicott J, Spitzer RL, Fleiss JL, Cohen J. The Global Assessment Scale: a procedure for measuring overall severity of psychiatric disturbance. Arch Gen Psychiatry 1976; 33: 766-71.

25. Drake RE, Mueser KT, McHugo GJ. Clinician rating scales: Alcohol Use Scale (AUS), Drug Use Scale (DUS) and Substance Abuse Treatment Scale (SATS). In: Sederer L, \& Dickey B, editors. In: Outcomes assessment in clinical practice. Baltimore: Williams and Wilkins, 1996, pp. 113-6.

26. Carrà G, Crocamo C, Borrelli $\mathrm{P}$, et al. Correlates of dependence and treatment for substance use among people with comorbid severe mental and substance use disorders: findings from the "Psychiatric and Addictive Dual Disorder in Italy (PADDI)" Study. Compr Psychiatry. 2015; 58: 152-9.

27. Carrà G, Johnson S, Crocamo C, et al. Psychosocial functioning, quality of life and clinical correlates of comorbid alcohol and drug dependence syndromes in people with schizophrenia across Europe. Psychiatry Res 2016; 239: 301-7. 
28. Tukey JW. Exploratory Data Analysis. Reading, MA: Addison-Wesley; 1977.

29. Thorup A, Petersen L, Jeppesen P, et al. Gender differences in young adults with firstepisode schizophrenia spectrum disorders at baseline in the Danish OPUS study. $J$ Nerv Ment Dis 2007; 195: 396-405.

30. Kraan T, Velthorst E, Koenders L, et al. Cannabis use and transition to psychosis in individuals at ultra-high risk: review and meta-analysis. Psychol Med 2016; 46: 673-81.

31. Del Vecchio V, Luciano M, Sampogna G, et al. The role of relatives in pathways to care of patients with a first episode of psychosis. Int J Soc Psychiatry. 2015; 61: 631-7.

32. Corrigan PW. Lessons learned from unintended consequences about erasing the stigma of mental illness. World Psychiatry. 2016; 15: 67-73.

33. Schomerus G, Angermeyer MC, Baumeister SE, Stolzenburg S, Link BG, Phelan JC. An online intervention using information on the mental health-mental illness continuum to reduce stigma. Eur Psychiatry. 2016; 32: 21-7.

34. Singh SP, Tuomainen H. Transition from child to adult mental health services: needs, barriers, experiences and new models of care. World Psychiatry. 2015; 14: 358-61.

35. Green L. Network Correlates of Social and Emotional Loneliness in Young and Older Adults. Personality and Social Psychology Bulletin 2001; 27: 281-8.

36. Bartoli F, Carrà G, Crocamo C, Carretta D, Clerici M. Bipolar disorder, schizophrenia, and metabolic syndrome. Am J Psychiatry. 2013; 170: 927-8.

37. Lloyd-Evans B, Crosby M, Stockton S, et al. Initiatives to shorten duration of untreated psychosis: systematic review. Br J Psychiatry 2011; 198: 256-63.

Table 1. Socio-demographic, clinical and social network characteristics

\begin{tabular}{|l|c|c|}
\hline & \multicolumn{2}{|c|}{ Total (N=122) } \\
\hline Age (yrs.), mean (SD) & $24.2(4.2)$ & \\
\hline Gender: Male & $82(67.2 \%)$ & \\
\hline Ethnicity & $50(41 \%)$ & \\
White UK and European & & \\
\hline
\end{tabular}

This article is protected by copyright. All rights reserved. 


\begin{tabular}{|l|c|c|}
\hline Black and Mixed black & $46(38 \%)$ & \\
Asian & $11(9 \%)$ & \\
Other & $15(12 \%)$ & \\
\hline Not in a relationship & $115(94.3 \%)$ & \\
\hline Not living alone & $66(54.1 \%)$ & \\
\hline Education (yrs.), mean (SD) & $12.5(3)$ & \\
\hline Unemployed & $90(74 \%)$ & \\
\hline Cannabis & $69(56.6 \%)$ & \\
\hline Alcohol & $93(76.2 \%)$ & \\
\hline DUP (mths), mean (SD) & $9.2(11.9)$ & \\
\hline GAF- S, mean (SD) & $36.7(16.9)$ & \\
GAF-D, mean (SD) & $45.3(17.1)$ & \\
\hline PANSS, mean (SD) & & \\
Total score & $69.0(25.0)$ & \\
Positive & $22.7(6.4)$ & \\
Negative & $18.5(8.6)$ & \\
General & $36.0(12.9)$ & \\
\hline Social network mean(SD) & Mean (SD) & Median (IQR) \\
\hline Total contacts & $11(15.5)$ & $7(5-11)$ \\
$\quad$ previous mth) & & \\
Relatives & $4.2(3.0)$ & $4(2-6)$ \\
Social acquaintances & $4.6(8.2)$ & $3(1-5)$ \\
Workmates & $1.5(12.1)$ & 0 \\
Confidants & $5(4.0)$ & $5(2-7)$ \\
With whom upset and in conflict & $1.6(2.9)$ & $1(0-2)$ \\
Would miss support & $7(7.5)$ & $5(3-8)$ \\
\hline Frequency & $4(5.9)$ & $2.5(1-5)$ \\
4 days/wk - weekly & $5(13.5)$ & $3(2-5)$ \\
3 days/wk - weekly & $2.5(5.1)$ & $1(0-3)$ \\
Monthly & $0.7(1.3)$ & $0(0-1)$ \\
Less than monthly & & \\
\hline SD, Sar & & \\
\hline
\end{tabular}

SD, Standard Deviation; IQR= Interquartile Range. DUP, Duration of Untreated Psychosis. Global Assessment of Functioning for Symptoms (GAF-S) and Disability (GAF-D).

PANSS, Positive And Negative Symptoms Scale. 
Table 2. DUP*, Number of confidants, and cannabis misuse: multiple linear regression model

\begin{tabular}{|l|c|c|c|c|c|c|}
\hline \multicolumn{1}{|c|}{ Variable } & Coeff. & SE & t & $\boldsymbol{p}$ & \multicolumn{2}{c|}{ 95\% CI. } \\
\hline Number of confidants & -.089 & .038 & -2.36 & 0.020 & -.164 & -.014 \\
\hline Cannabis misuse & .435 & .275 & -1.58 & 0.116 & .980 & .109 \\
\hline constant & 2.080 & .261 & 7.98 & $<0.001$ & 1.563 & 2.596 \\
\hline
\end{tabular}

* $\log$ transformed DUP (mths); DUP, Duration of Untreated Psychosis. SE, Standard Error. CI, Confidence Interval. 


\section{University Library}

\section{- M I N E R VA A gateway to Melbourne's research publications}

Minerva Access is the Institutional Repository of The University of Melbourne

Author/s:

Carra, G;Battaglia, C;Hinton, M;Rains, LS;Crocamo, C;Johnson, S

Title:

Social network confidants, duration of untreated psychosis and cannabis use in people with first episode psychosis: An exploratory study

Date:

2018-10-01

Citation:

Carra, G., Battaglia, C., Hinton, M., Rains, L. S., Crocamo, C. \& Johnson, S. (2018). Social network confidants, duration of untreated psychosis and cannabis use in people with first episode psychosis: An exploratory study. EARLY INTERVENTION IN PSYCHIATRY, 12 (5), pp.942-946. https://doi.org/10.1111/eip.12476.

Persistent Link:

http://hdl.handle.net/11343/293391 